\title{
Examining the Possibilities and Pitfalls of Three Dimensional Energy Filtered Transmission Electron Microscopy (3D-EFTEM)
}

\author{
M.Weyland and P. A. Midgley \\ Department of Materials Science and Metallurgy, University of Cambridge, Pembroke Street, Cambridge, \\ CB2 3QZ UK
}

The last three years has seen a rapid growth in interest in carrying out electron tomography, a technique previously dominated by the biosciences, in materials science. The demands of inorganic materials, so different to those of the biological, has lead to a range of new approaches being applied: particular success has been achieved in HAADF STEM tomography [1]. While several groups have also demonstrated that EFTEM tomography is possible [2, 3] there are many uncertainties to the validity and best practice of the technique.

The basic requirement of tomography is that any two-dimensional (2D) image used for reconstruction must show contrast that is at least monotonic with thickness [4]. However both EFTEM jump-ratio images and elemental maps can, under certain conditions, show a nonmonotonic relationship to thickness. In jump-ratio images the intensity observed shows a monotonic relationship only when the thickness is under approximately $t / \lambda=0.8-1.0$, as shown in FIG. 1 . Therefore for thicker specimens jump-ratio images may produce misleading reconstructions, an effect that may be amplified by the increase in sample thickness at the high tilts tomography demands. Elemental maps however, show deviations from monotonic contrast due to the effects of residual diffraction contrast, as shown in FIG. 2 (note however the lack of diffraction effects in the jump-ratio image). These two factors would seem to preclude the use of EFTEM elemental distribution images from tomographic reconstruction. However, results have shown that reconstruction can be achieved, and they can reveal the distinctive $3 \mathrm{D}$ nature of elemental distribution in specimens [2,3]. It has been shown that jump-ratio maps can be used for reconstruction for thin specimens, especially those dispersed on carbon grids, where the specimen thickness stays below $t / \lambda=1$.0. It has also proved possible to reconstruct from elemental maps, even from heavily diffracting specimens [3], as diffraction effects tend to average out over the range of tilt, in a way similar to that observed when using conical or rocking beam illumination [5].

To date all EFTEM tomographic reconstruction has been carried out using processing the energyloss tilt series to calculate a single tilt series of maps, by division or $\mathrm{AE}^{-\mathrm{r}}$ extrapolation. This process is shown schematically as "Route 1" in FIG. 3. However it is also possible to reverse this approach and reconstruct each energy-loss series individually giving "energy-loss" volumes that can then by processed, voxel-by-voxel, to produce a 3D elemental map, as in "Route 2" in FIG. 3. This proposed route effectively generates a $4 \mathrm{D}$ data space $(\Delta \mathrm{E}, \mathrm{x}, \mathrm{y}, \mathrm{z})$ that should, especially if extended beyond three images, allow at least semi-quantitative analysis of 3D elemental maps.

[1] M. Weyland et al., Journal of Physical Chemistry B 105 (33) (2001) 7882.

[2] G. Mobus et al., Applied Physics Letters 79 (9) (2001) 1369.

[3] M. Weyland et al., Institute of Physics Conference Series 161(EMAG 2001) (2001) 239.

[4] P.W. Hawkes, in Electron tomography : three-dimensional imaging with the transmission electron microscope, J. Frank, (Editor), Plenum Press, New York ; London, 1992 p. 17.

[5] P. Warbichler et al., Micron 29 (1) (1998) 63. 


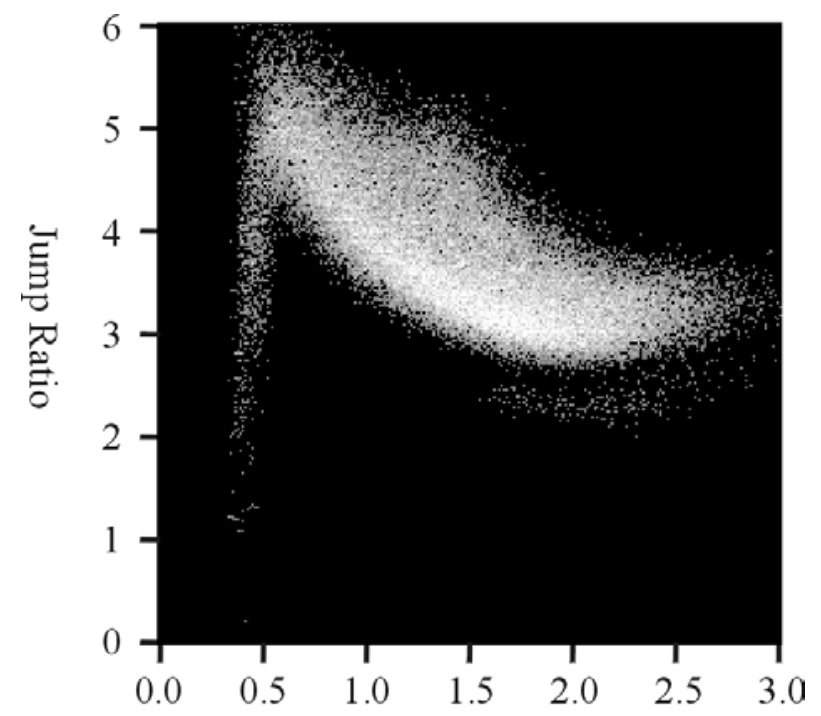

FIG. 1. An experimental plot of jump-ratio vs. $t / \lambda$ for a $C r$ wedge specimen. For values of $t / \lambda$ up to about 0.8 the graph is monotonic, yet increasing thickness lowers the jump-ratio: invalidating the projection requirement. (Plot courtesy of Dr P.Thomas.)

Original (aligned) data

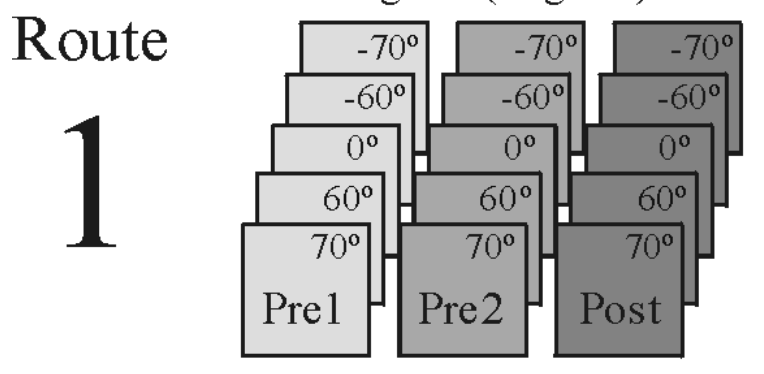

Original (aligned) data
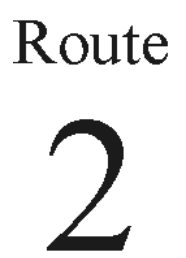

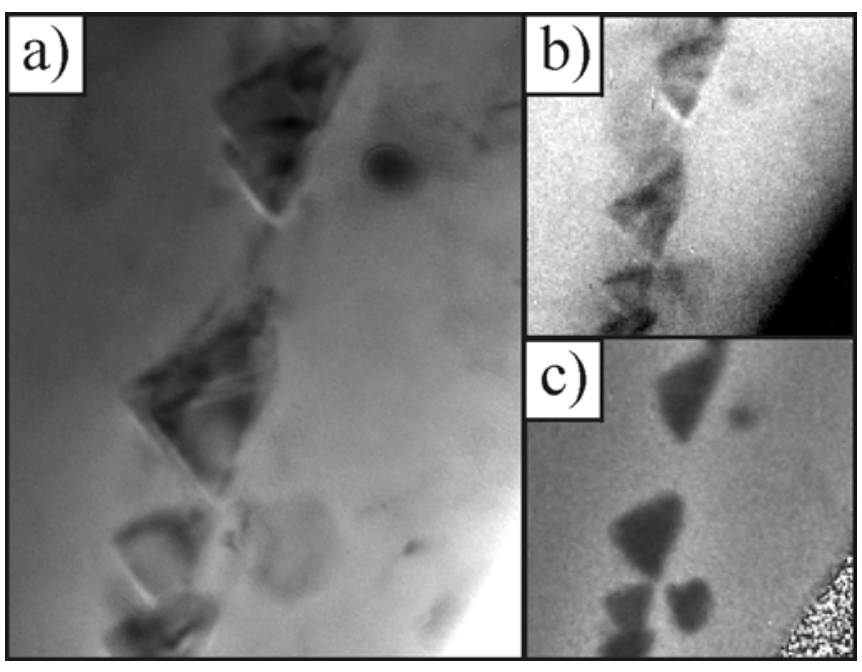

FIG. 2. An example of residual diffraction contrast. a) A Zeroloss image of $\mathrm{Cr}$ carbides in a stainless steel. b) Elemental map showing residual diffraction contrast and c) jump-ratio showing removal of diffraction effects. Both maps generated from the $\mathrm{Fe}_{23}$ edge.

Tilt series of maps

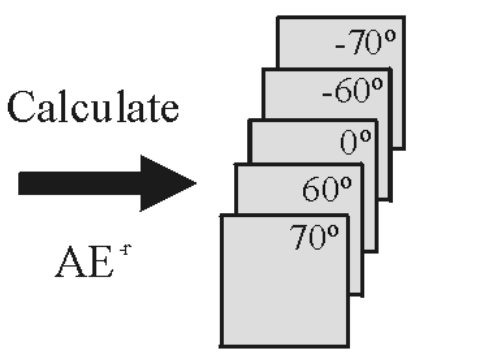

\section{Tomographic} reconstruction
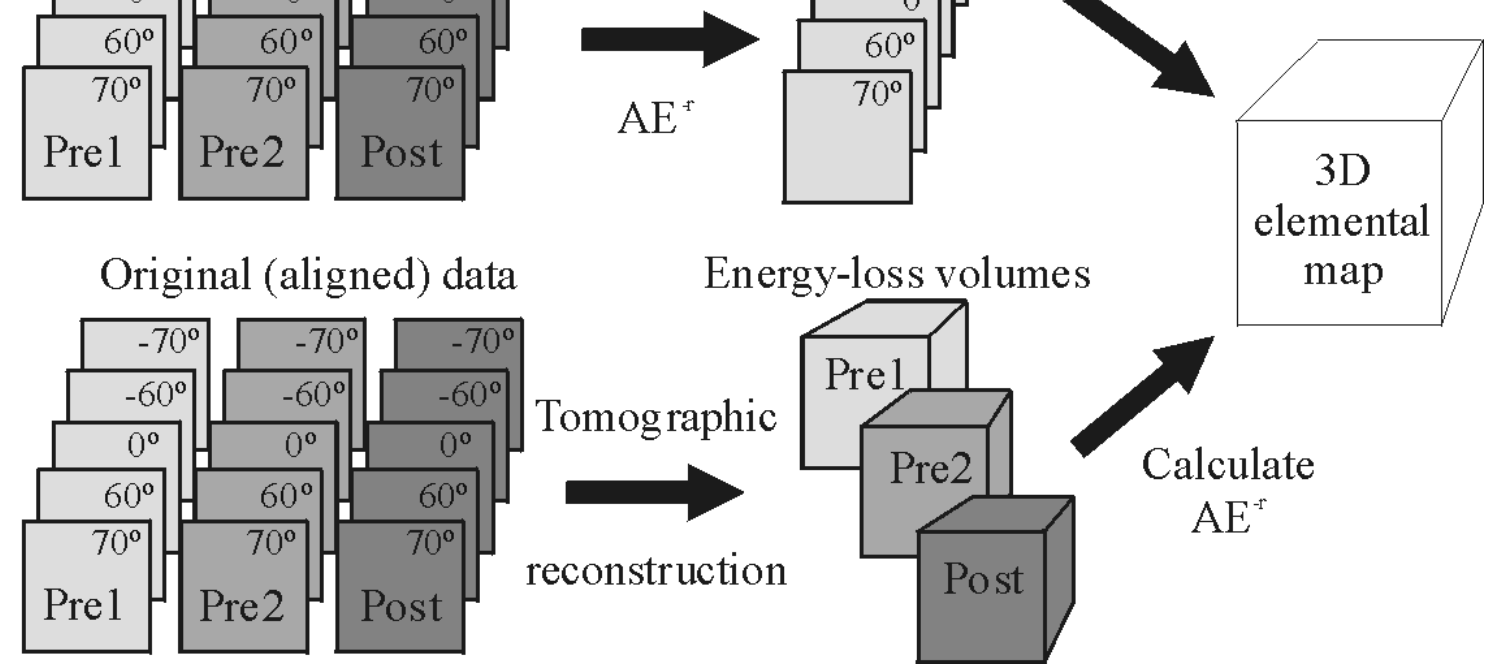

Calculate

$\mathrm{AE}^{-\mathrm{r}}$

FIG. 3. A schematic of the two possible ways of producing a three dimensional elemental map, different only in the order which the operations are carried out. In route 1 the aligned data is first reduced to a single tilt series of maps from which a 3D map is reconstructed. In route 2 several energy-loss volumes are generated by reconstructing individual energy-loss tilt series. The second may allow more accurate generation and quantification of 3D elemental maps. 